\title{
Optical Evaluation of a Refractive Secondary Concentrator
}

Donald A. Jaworske and Wayne A. Wong

Glenn Research Center, Cleveland, Ohio

Timothy J. Skowronski

Cleveland State University, Cleveland, Ohio

Prepared for the

34th Intersociety Energy Conversion Engineering Conference sponsored by the Society of Automotive Engineers

Vancouver, British Columbia, Canada, August 1-5, 1999

National Aeronautics and

Space Administration

Glenn Research Center 


\section{Acknowledgments}

The authors would like to thank Bob Macosko, Don Buchele, and Charlie Castle for their helpful discussions on refractive secondary concentrators.

Available from

NASA Center for Aerospace Information

7121 Standard Drive

Hanover, MD 21076

Price Code: A03
National Technical Information Service 5285 Port Royal Road Springfield, VA 22100 Price Code: A03 


\title{
Optical Evaluation of a Refractive Secondary Concentrator
}

\author{
Donald A. Jaworske and Wayne A. Wong \\ NASA Glenn Research Center \\ Cleveland, Ohio 44135 \\ Timothy J. Skowronski \\ Cleveland State University \\ Cleveland, Ohio 44121
}

\begin{abstract}
Refractive secondary concentrators are being considered for solar thermal applications because of their ability to achieve maximum efficiency through the use of total internal reflection for the concentration and distribution of solar energy. A prototype refractive secondary concentrator was built based on ray tracing analysis to demonstrate this collection and distribution concept. The design included a conical secondary concentrator and a faceted extractor. The objective of this effort was to functionally evaluate the performance of the refractive secondary concentrator/extractor prototype and to compare the results with modeling. Most of the light was found to exit the refractive secondary concentrator through the extractor. In addition, the degree of attenuation encountered by the light as it passed through the refractive secondary concentrator was of interest. Quantifying optical output and validating the modeling will provide further understanding of the efficiency of the prototype and will provide insight for additional design and materials selection activities.
\end{abstract}

\section{INTRODUCTION}

In a typical solar thermal application, the optical pathway plays the key role of delivering energy to the heat receiver. Energy must be delivered to the receiver with a minimum of reflection and absorption losses, and the energy must be directed into the receiver through the smallest possible opening to minimize infrared radiation losses. Minimizing these losses maximizes system efficiency. The refractive secondary concentrator provides one means of maximizing energy delivered to the heat receiver while minimizing energy loss.

The refractive secondary concentrator utilizes a crystal machined to the desired shape. This crystal provides a total internal reflection pathway that concentrates and directs sunlight into a flux extractor, which then distributes the energy in the receiver. Unlike a reflective secondary concentrator, where light is reflected by a parabolic mirror at some reflectance loss, total internal reflection yields no energy loss at the internal point of reflection. Some reflection loss may occur at the surface. The shape of the crystal and design of the extractor defines where in the cavity the concentrated sunlight is delivered. Crystals that survive high temperature are needed to withstand the temperatures inside the receiver and crystals that are transparent are needed to minimize absorption.

Much work has been done to support the design and development of refractive secondary concentrators. ${ }^{1-4}$ Briefly, a ray tracing program was used to iterate the 3-dimensional shape and contour of an idealized refractive secondary concentrator and extractor. The original design using a dielectric totally internally reflecting concentrator ${ }^{3}$ evolved into a cone and extractor combination. An acrylic prototype of this configuration was manufactured at NASA Glenn Research Center for testing, as part of the Shooting Star program sponsored by NASAMarshall Space Flight Center. ${ }^{1}$ The purpose of this paper is to functionally evaluate the performance of the refractive secondary concentrator/extractor prototype and to compare those results with modeling. Studies on the attenuation of light as it passes through the refractive secondary concentrator are of continuing interest.

\section{MATERIALS AND METHODS}

Figure 1 summarizes the geometry of the acrylic prototype used in this study and defines its two main regions, a conical region and a faceted extractor. Specific details of the refractive secondary concentrator design are summarized in a previous study. ${ }^{1}$ The index of refraction was $1.49 .^{1}$

The conical region provides a means to collect rays within a half-angle of $22^{\circ}$ while the extractor provides a means to efficiently extract the energy and tailor the flux on the interior walls of the cavity, one of the key benefits of the refractive secondary concentrator. 


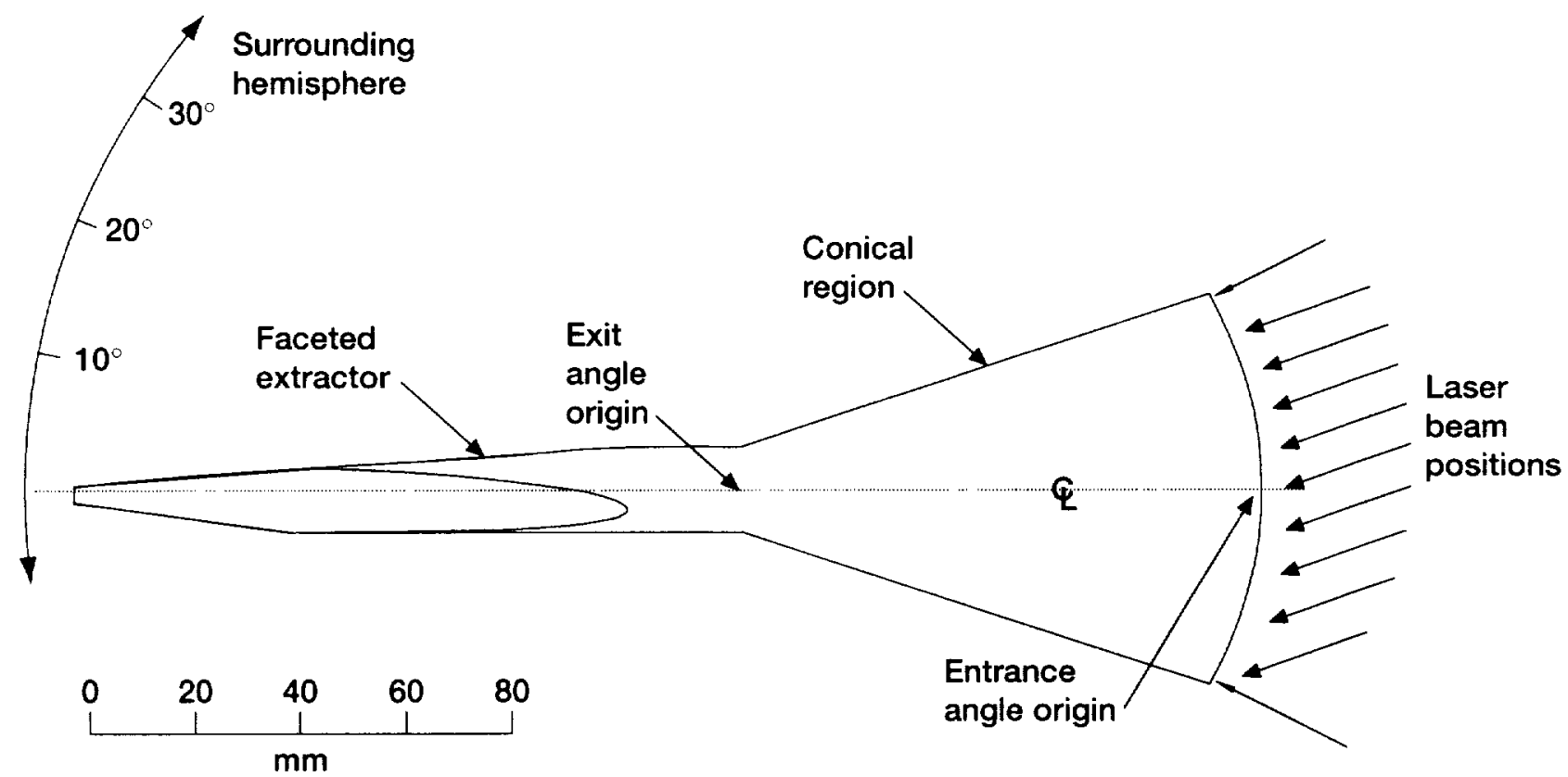

Figure 1.-Schematic diagram of the refractive secondary concentrator with entrance angle origin and exit angle origin defined.

In this study, the set up on the optical bench consisted of a chopped helium-neon laser beam directed onto a half silvered mirror that split the beam into a sample and reference beam. The sample beam was allowed to impinge on the refractive secondary concentrator from a variety of entrance angles and positions, while the reference beam was simultaneously directed into a reference detector. The reference detector was used to gauge the amount of drift in the laser over the duration of the experiment. The sample detector was moved through a series of discrete positions surrounding the exit of the refractive secondary concentrator to detect sample signal strength. Two coordinate systems were established, one centered around the front of the conical region to identify laser beam input and one centered around the exit of the conical region to identify laser beam output. These two coordinate systems were similar to coordinate systems used in a previous study. ${ }^{5}$ The coordinate system is identified in Figure 1.

The discrete positions at the exit of the refractive secondary concentrator were defined by the intersections of lines of latitude and longitude on an imaginary hemisphere surrounding the refractive secondary concentrator, separated by $10^{\circ}$ increments. The origin of the hemisphere was the defined exit angle origin and the radius of the hemisphere was $22.6 \mathrm{~cm}$. Part of the hemisphere cross section is shown schematically in Figure 1 . The radius of the hemisphere was chosen so as to enclose the extractor. The $1 \mathrm{~cm} \times 1 \mathrm{~cm}$ silicon detector was placed at the intersections of the lines of latitude and longitude. Given the $22.6 \mathrm{~cm}$ radius of the hemisphere and the $1 \mathrm{~cm}^{2}$ area of the silicon detector, it should be noted that the area collectively occupied by the detector is less than the area of the hemisphere.

Intensity data as a function of detector position, for a given entrance angle and position, were collected by moving the detector to each intersection, in sequence. This task was accomplished by bolting the detector to a suitable framework. Intensity data were recorded at each azimuth and elevation value, based on the exit origin of the refractive secondary concentrator. Once an array of intensity versus azimuth and elevation had been obtained, the data were plotted in a 3-dimensional graph. The process was repeated for the next entrance angle or position.

For the purpose of screening, nine equidistant positions were evaluated, by moving the laser beam at a fixed angle of $20^{\circ}$ across the diameter of the refractive secondary concentrator as shown schematically in Figure 1. Note that the laser beam was placed within a plane which also contained the axis of the refractive secondary concentrator. From these screening studies, one specific case was selected for comprehensive evaluation and comparison to modeling. For the comprehensive evaluation, intensity data were collected at discrete positions defined by lines of latitude and longitude separated by $5^{\circ}$. In this way, the output was recorded with greater resolution for comparison with modeling. 
The screening study $\left(10^{\circ}\right)$ and the one specific case for comprehensive evaluation $\left(5^{\circ}\right)$ mentioned above all represent meridional rays, rays that lie within a plane which also contains the axis of the refractive secondary concentrator. An additional case was evaluated, the skew ray case, to represent rays that do not lie in the same plane as the meridional rays. This was accomplished by selecting a skew ray at random. Again, output was recorded at $5^{\circ}$ increments to yield greater resolution for comparison with modeling.

In addition to the optics bench activities, diamond-turned disks of acrylic, $2.54 \mathrm{~cm}$ in diameter and of various thickness, were used to evaluate the attenuation of light. Various thickness disks were selected to do the evaluation analogous to the method of successive additions. Transmittance measurements were made on the acrylic disks in the wavelength range of 250 to $2500 \mathrm{~nm}$ using a Perkin-Elmer Lambda-19 spectrophotometer equipped with a $15 \mathrm{~cm}$ diameter integrating sphere. Absorptance and reflectance losses were calculated at the wavelength of the HeNe laser, $632 \mathrm{~nm}$.

One acrylic disk was placed in the center of the $15 \mathrm{~cm}$ diameter integrating sphere by using a center mount attachment. In this way, combined transmittance and reflectance values were obtained over the wavelength range of 250 to $2500 \mathrm{~nm}$.

The acrylic refractive secondary concentrator was modeled using a ray trace program. ${ }^{4}$ Figure $2 a$ illustrates a ray trace through the refractive secondary concentrator using as input random rays within the designed half-angle of $22^{\circ}$. The plot in figure $2 \mathrm{~b}$ shows the resulting variation of flux intensity along the length of a surrounding cylindrical cavity. By design, most of the flux impinges towards the rear of the cavity.

Modeling results showing the flux intensity exiting the refractive secondary concentrator are shown in figure $2 \mathrm{c}$. The flux intensity map is projected onto a hemispherical surface beyond the tip of the extractor analogous to the hemisphere shown schematically in Figure 1. The majority of the energy entering the refractive secondary concentrator as random rays strikes this surface within a distinct radius equivalent to a half-angle of approximately $30^{\circ}$.

Of the two comprehensive cases mentioned above, the skew ray condition is summarized in the flux intensity map shown in Figure $2 \mathrm{~d}$. The laser beam was modeled as a bundle of many parallel rays of comparable cross section. This bundle of rays was set up in the model to represent the same random orientation of the laser beam as found in the skew ray evaluation.

\section{RESULTS AND DISCUSSION}

Monitoring the intensity of the HeNe laser at the reference detector revealed that there was essentially no drift in the laser over the course of data collection. Hence, intensity at the sample detector is reported in millivolts.

Figure 3 summarizes the intensity data from the screening study, plotted as a function of azimuth and elevation. The input was meridional, at an entrance angle of $20^{\circ}$ and at nine equidistant positions across the diameter of the refractive secondary concentrator, starting near one edge and progressing across to the other edge. The interesting feature to notice in this series is that most of the light exits the refractive secondary concentrator in the vicinity of $\pm 30^{\circ}$. This is qualitatively similar to the rays exiting the refractive secondary concentrator model shown in Figure 2. The series also shows that the light detected upon exiting the refractive secondary concentrator is detected first in a single peak and progresses through a dual peak transition to a second single peak.

The comprehensive meridional ray case, shown in Figure 4a, was similar. Again light was introduced to the refractive secondary concentrator at an entrance angle of $20^{\circ}$. This time, the refractive secondary concentrator was placed such that the bottom edge of the triangular extractor was approximately parallel to the bench top. Most of the light exiting the refractive secondary concentrator was contained within one peak. Two subtle peaks were just noticeable at equilateral positions. The equilateral positions coincided with the equilateral edges of the extractor.

The comprehensive skew ray case is shown in Figure 4b. Again the refractive secondary concentrator was placed such that the bottom edge of the triangular extractor was approximately parallel to the bench top. Unlike the meridional ray case, the skew ray case yielded a pattern containing many peaks. Notice that the maximum intensity of the peaks decreased substantially from the meridional ray case. All else being equal, the cumulative area under all the peaks in Figure $4 \mathrm{~b}$ should approximate the area under the three peaks in Figure $4 a$. In the comprehensive skew ray case, most of the light exiting the refractive secondary concentrator was from the faces of the extractor, not the edges. Again, most of the light exits the refractive secondary concentrator in the vicinity of $\pm 30^{\circ}$, qualitatively similar to the rays exiting the refractive secondary concentrator model shown in Figure 2.

Closer comparison between Figure $2 \mathrm{~d}$, the intensity map produced from modeling the skew ray case and Figure $4 b$, the intensity map resulting from the bench top 


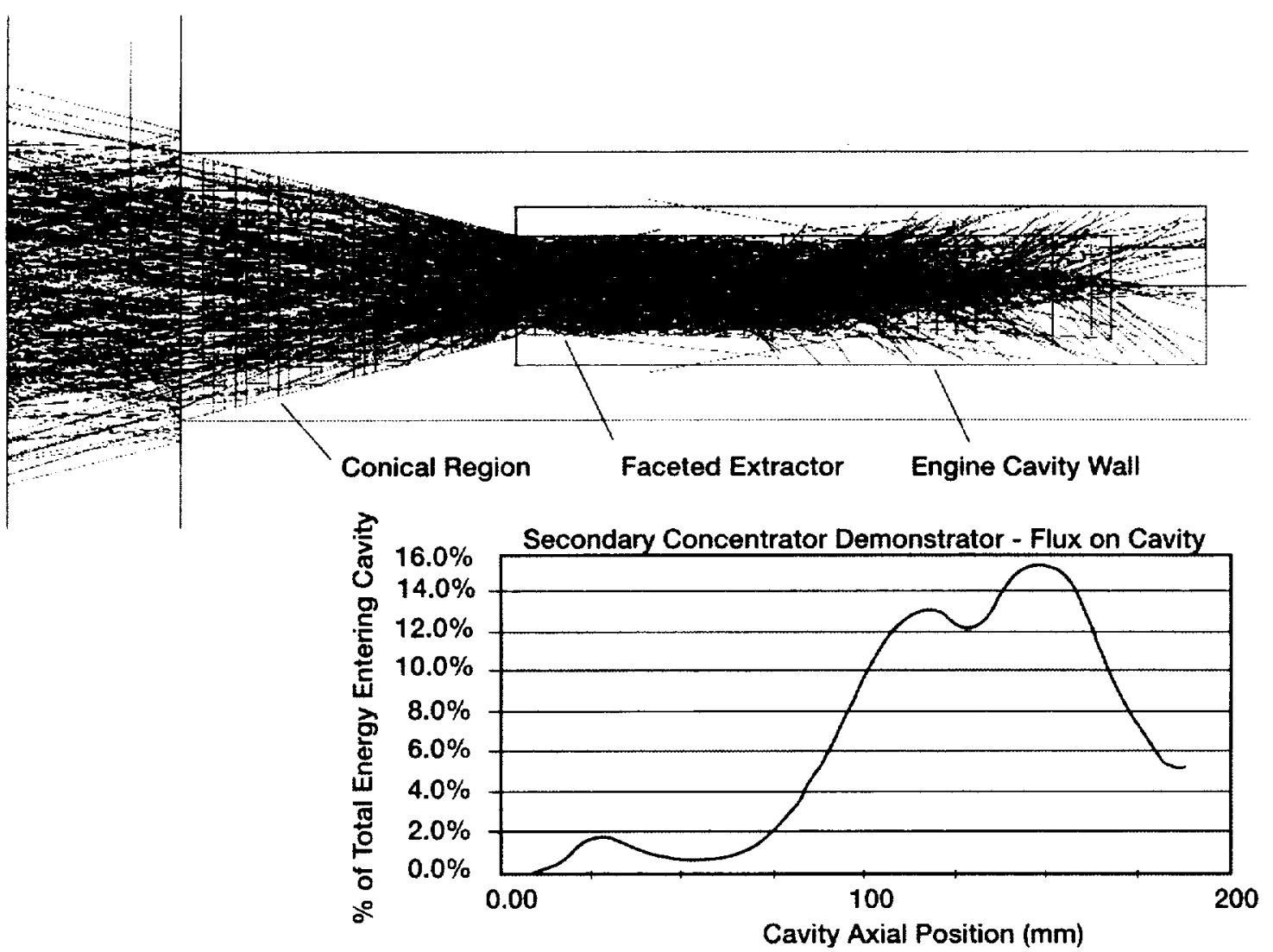

(a)

(b)

12

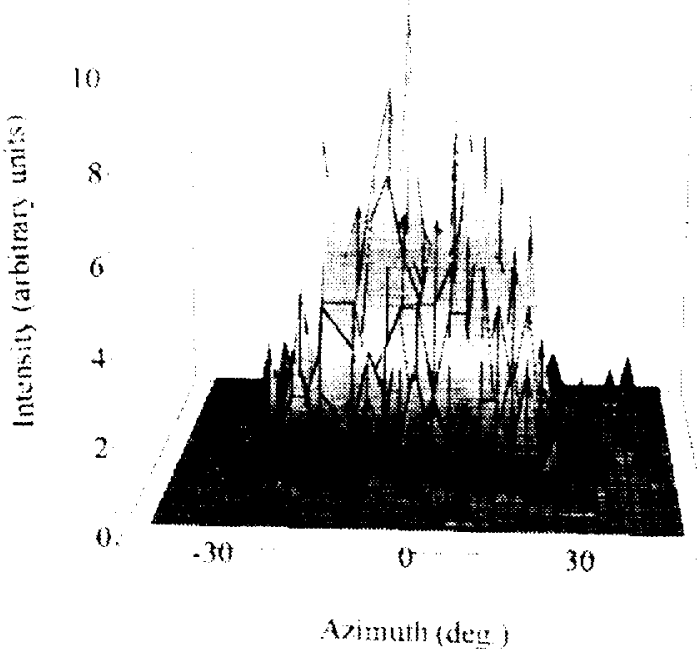

(c)

80

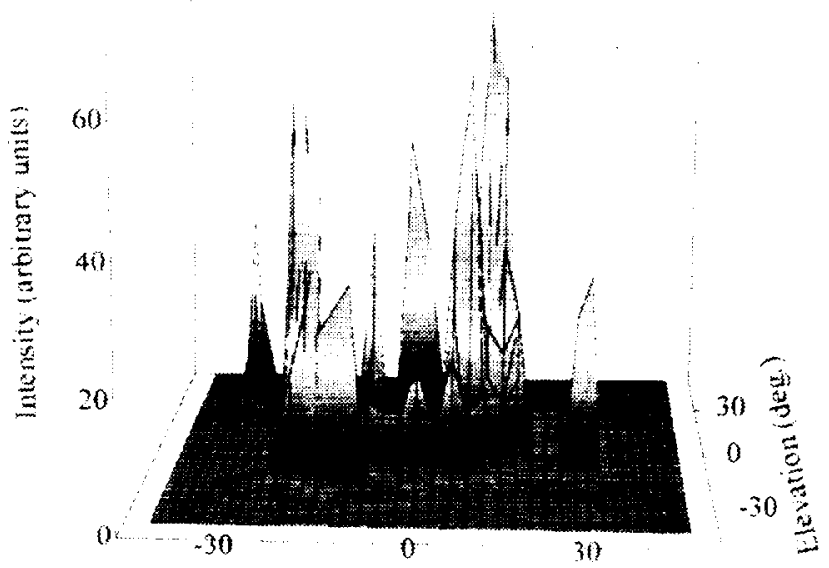

Aximuth (deg. )

(d)

Figure 2.-Flux projected by ray tracing showing (a) random rays entering within a 22 degree half-angle, (b) flux distribution along the cavity wall, (c) intensity map of exiting flux for the model case using random input rays and (d) intensity map of exiting flux for the model case using a bundle of skew rays. 

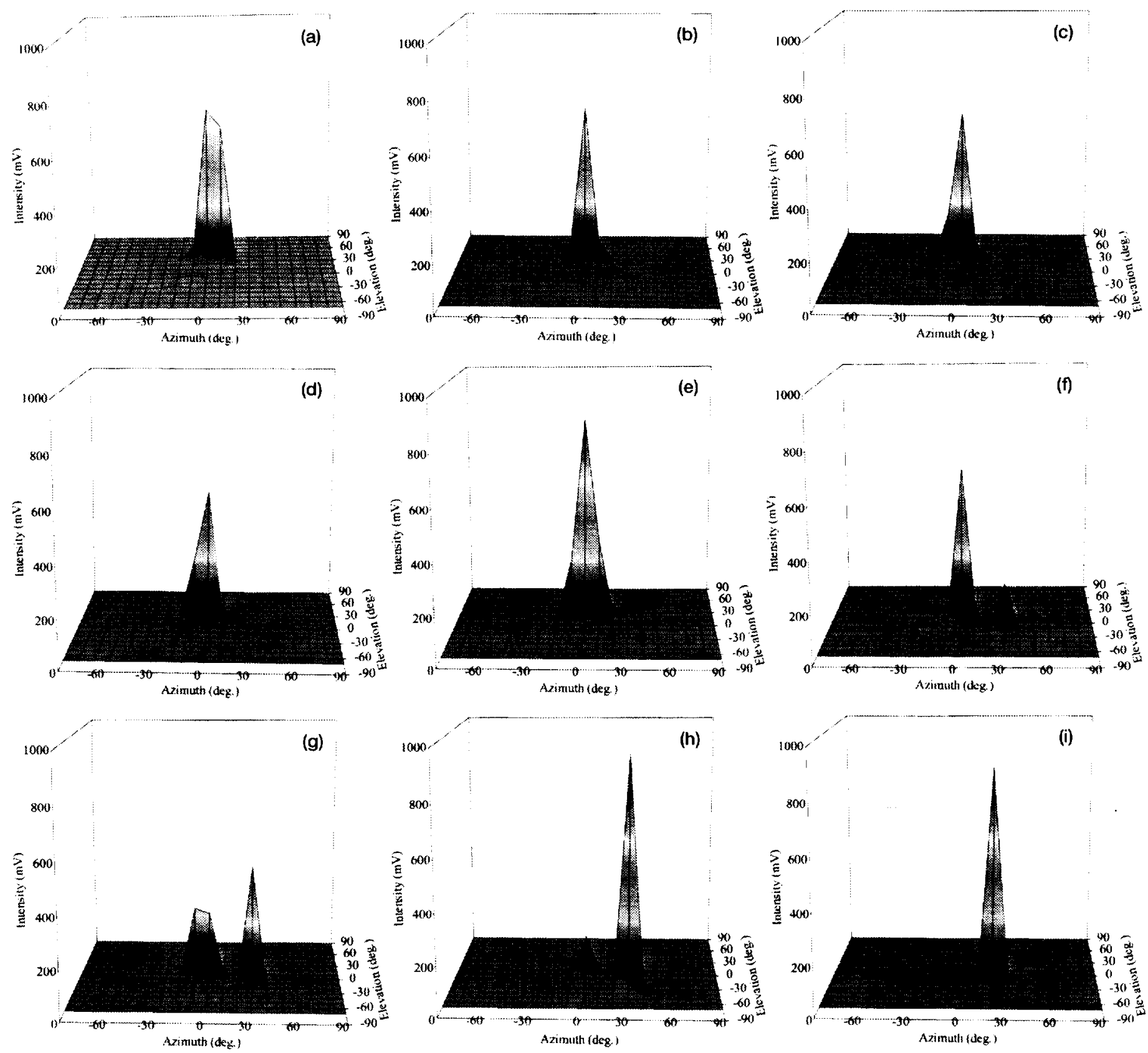

Figure 3.-Exit intensity as a function of azimuth and elevation for an entrance angle of $20^{\circ}$ and translation positions of (a) 0 , (b) $6.3 \mathrm{~mm}$, (c) $12.7 \mathrm{~mm}$, (d) $19.05 \mathrm{~mm}$, (e) $25.4 \mathrm{~mm}$, (f) $31.75 \mathrm{~mm}$, (g) $38.10 \mathrm{~mm}$, (h) $44.45 \mathrm{~mm}$ and (i) $50.8 \mathrm{~mm}$. 

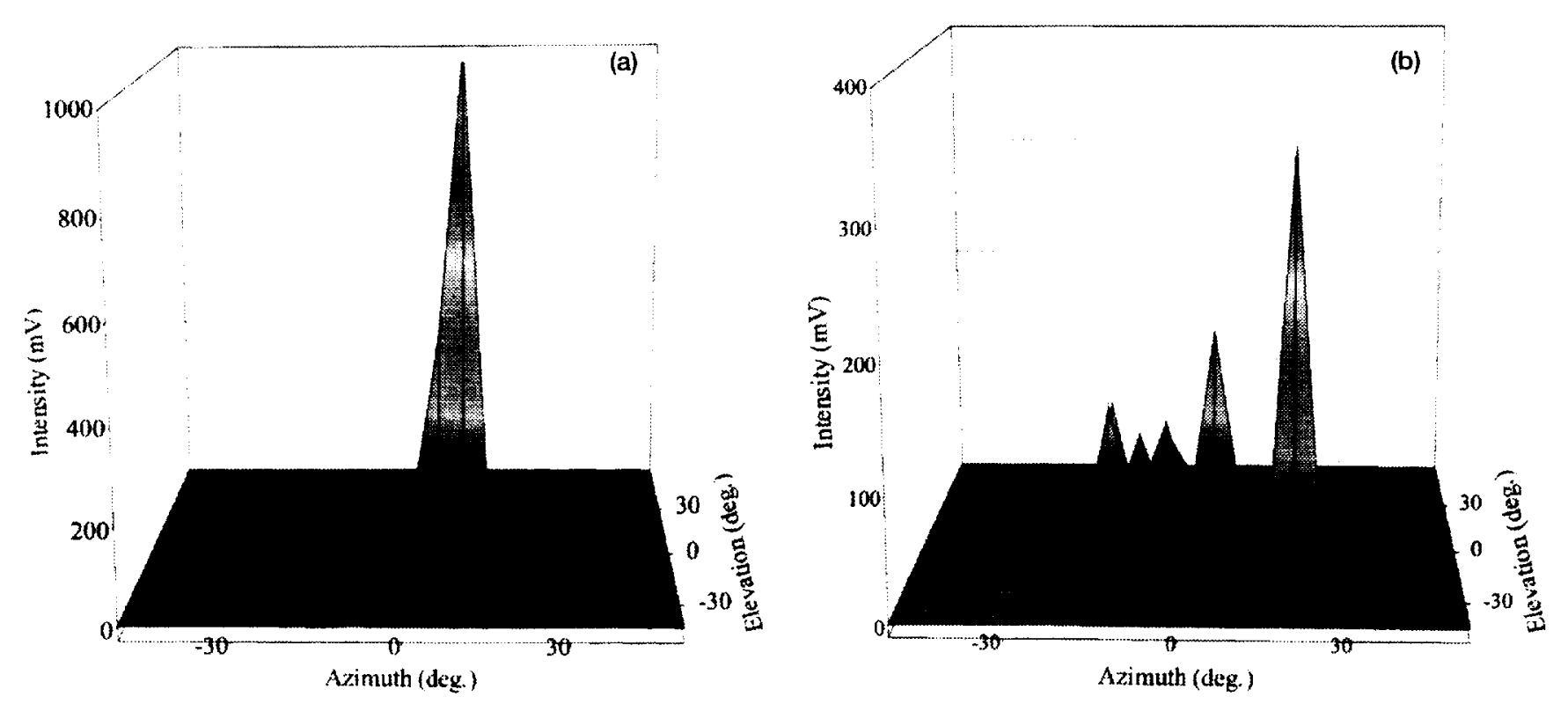

Figure 4.-Exit intensity for (a) the comprehensive meridional ray case and (b) the comprehesive skew ray case, as a function of azimuth and elevation.

skew laser beam evaluation reveals that there is a remarkable correlation between the model and the test results. Both intensity maps exhibit a similar shape and have high peaks in generally the same locations relative to the exit angle origin of the refractive secondary concentrator.

A discussion of the ratio of meridional rays versus skew rays occurring from a given primary concentrator light source is beyond the scope of this paper. However, experience would suggest that the population of skew rays dominates the population of meridional rays. Understanding the impact of the primary concentrator light source, on the output of the refractive secondary concentrator, including the dominant population of skew rays, will have to rely on further modeling and performance testing. A discussion of the implication of utilizing materials with different indexes of refraction is appropriate. One high temperature material envisioned for use in actual hardware is sapphire. Sapphire has an index of refraction of 1.76, a value slightly higher than acrylic. ${ }^{1}$ For a cone of the same entrance diameter, the higher index of refraction would drive the exit diameter of the cone and the diameter of the extractor to a smaller value, increasing the aspect ratio of the extractor.

The results from the transmittance study of acrylic disks are summarized in Figure 5. In some regions of the spectrum, the transmittance varies as a function of thickness while in other regions, the transmittance does not vary as a function of thickness. At $632 \mathrm{~nm}$, the transmittance does not vary as a function of thickness, suggesting the absence of absorptance. Figure 6 shows this, by plotting transmittance at $632 \mathrm{~nm}$ versus sample thickness. Extrapolating to zero thickness yields the only loss, a reflectance loss of $7.4 \%$. This reflectance loss is from the face of the acrylic and is the same regardless of sample thickness.

To confirm the absence of absorptance at $632 \mathrm{~nm}$, one sample $6.3 \mathrm{~mm}$ thick was placed inside the integrating sphere of the spectrophotometer by utilizing a center mount attachment. By rotating the sample to an angle of approximately $30^{\circ}$, such that the specular portion reflecting off the diamond turned face is also captured by the

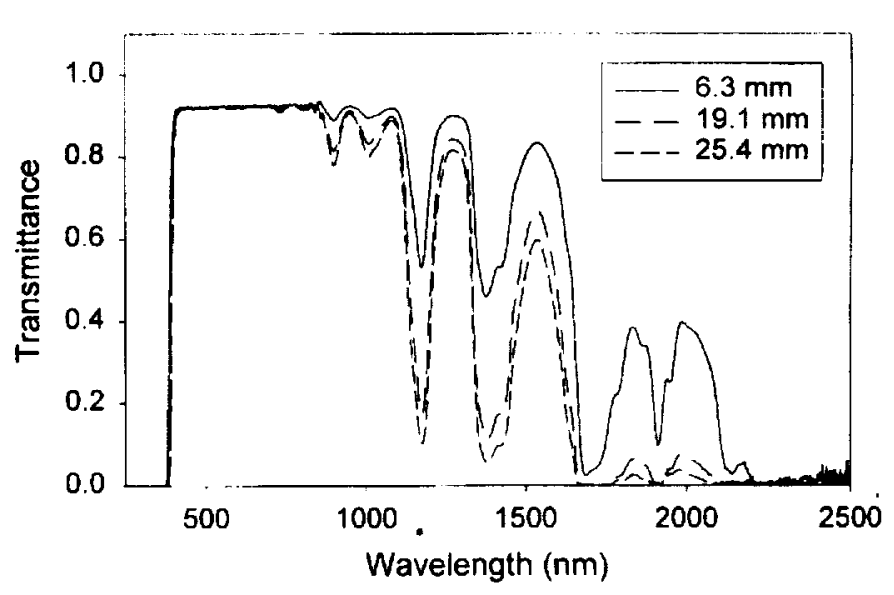

Figure 5.-Transmittance versus wavelength for three acrylic disks, $6.3,19.1$, and $25.4 \mathrm{~mm}$ thick. 


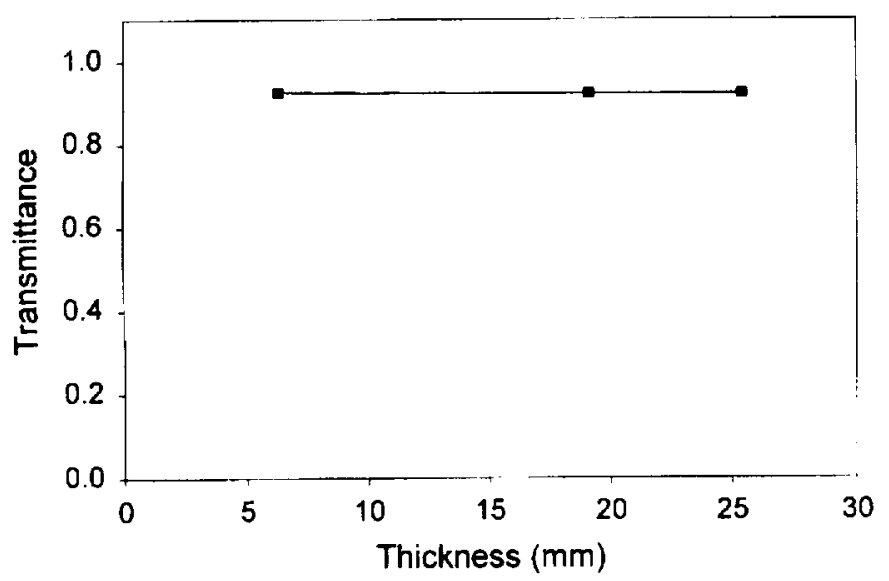

Figure 6.-Transmittance as a function of thickness, at $632 \mathrm{~nm}$.

sphere, it was possible to obtain the sum of transmittance and reflectance, leaving absorptance by difference. As shown in Figure 7, the sum of transmittance and reflectance at $632 \mathrm{~nm}$ was 1 , confirming that the absorptance was zero. It is interesting to note that the absorptance in other regions of the spectrum is not zero.

The absorptance of acrylic at $632 \mathrm{~nm}$ will be of interest for future energy balance evaluations. The acrylic sample also demonstrates the successful use of the center mount attachment for future attenuation studies of other materials of interest.

Although acrylic was used here, it is not suitable for high temperature applications. Transmittance, reflectance, and absorptance of candidate materials such as (in order of increasing application temperature) quartz, sapphire and zirconia will have to be evaluated in a similar fashion to identify reflection and absorption losses over the entire air mass zero solar spectrum.

\section{FUTURE WORK}

One idea for improving the technique to measure optical throughput is to utilize an integrating sphere at the exit of the refractive secondary concentrator. In this way, light from the exit is captured in its entirety. The integrating sphere would accept light from the refractive secondary concentrator after the laser beam has engaged the refractive secondary concentrator. It could also accept light without engaging the refractive secondary concentrator by introducing the laser beam to the integrating sphere through a different port. The refractive secondary concentrator must be mated to the integrating sphere during both types of measurements to account for energy entering and leaving the integrating sphere via transmission

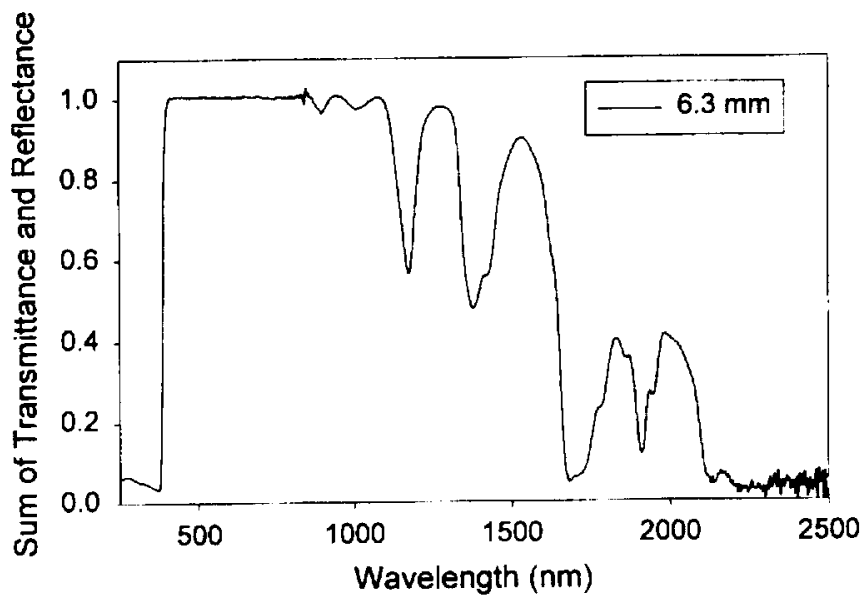

Figure 7.-Transmittance and reflectance combined versus wavelength, for a $6.3 \mathrm{~mm}$ thick acrylic disk, measured using a center mount integrating sphere attachment.

through the refractive secondary concentrator. The ratio of these two values would yield the optical throughput, or efficiency, of the refractive secondary concentrator. Ideally, one would like to use a converging light source analogous to a primary concentrator to illuminate a sector of the refractive secondary concentrator, or perhaps the entire refractive secondary concentrator.

\section{CONCLUSIONS}

A previously designed refractive secondary concentrator acrylic prototype was evaluated on an optical bench using a helium neon laser light source and variable position silicon detector. The purpose of the evaluation was to substantiate the modeling activities used to design the refractive secondary concentrator and to provide additional support in advocating the use of secondary concentrators in a variety of solar thermal applications.

Results of the bench top evaluation compare very favorably to the results from the ray trace model. Light was found to pass through the refractive secondary concentrator as expected for both meridional rays and skew rays. On a qualitative level, both the bench top evaluation and the modeling of random input rays illustrates that the energy exits the refractive secondary concentrator within a cone of approximately of $\pm 30^{\circ}$. Detailed comparison between the bench top results and the modeling for a random skew ray condition also shows excellent correlation.

Although the refractive secondary concentrator relies on total internal reflection to direct light to its desired exit, one issue that must be resolved is attenuation of the light due 
to reflection and absorption losses. To address this issue, selected acrylic disks were evaluated in a spectrophotometer to identify reflectance and absorptance losses. At a wavelength of $632 \mathrm{~nm}$, it was found that there was a $7.4 \%$ reflectance loss and no detectable absorptance loss. This observation was verified by placing an acrylic sample in the center mount attachment of the integrating sphere used to make transmittance, absorptance, and reflectance measurements. These losses will have to be revisited, and minimized, during the design and manufacture of secondary concentrators made from sapphire or other materials suitable for use at high temperatures.

\section{DEFINITIONS, ACRONYMS, ABBREVIATIONS}

Meridional ray: A ray that lies within a plane which also contains the axis of an optical system.

Skew ray: $A$ ray that does not lie in the same plane as the axis of an optical system.

\section{REFERENCES}

1. J. A. Soules, D. R. Buchele, C. Castle, and R. Macosko, "Design and Fabrication of a Dielectric Total Internal Reflecting Solar Concentrator and Associated Flux Extractor for Extreme High Temperature (2500K) Applications," NASA Contractor Report 204145, 1997.

2. D. Zhu, N. S. Jacobson, and R. A. Miller, "ThermalMechanical Stability of Single Crystal Oxide Refractive Concentrators for High-Temperature Solar Thermal Propulsion," ASME International Solar Energy Conference, Lahaina, Maui, HI, 1999.

3. X. Ning, R. Winston, and J. O'Gallagher, "Dielectrically totally internally reflecting concentrators," Applied Optics, Volume 26(2), pp. 300-305. 1987.

4. W. A. Wong and R. P. Macosko, "Refractive Secondary Concentrators for Solar Thermal Applications," IECEC1999-01-2678, Vancouver, BC, 1999.

5. D. A. Jaworske, T. J. Skowronski and B. J. Miles, "Optical Evaluation of an As-Manufactured Compound Secondary Concentrator," Space Technology \& Applications International Forum, Albuquerque, NM, pp 621-626, 1999. 


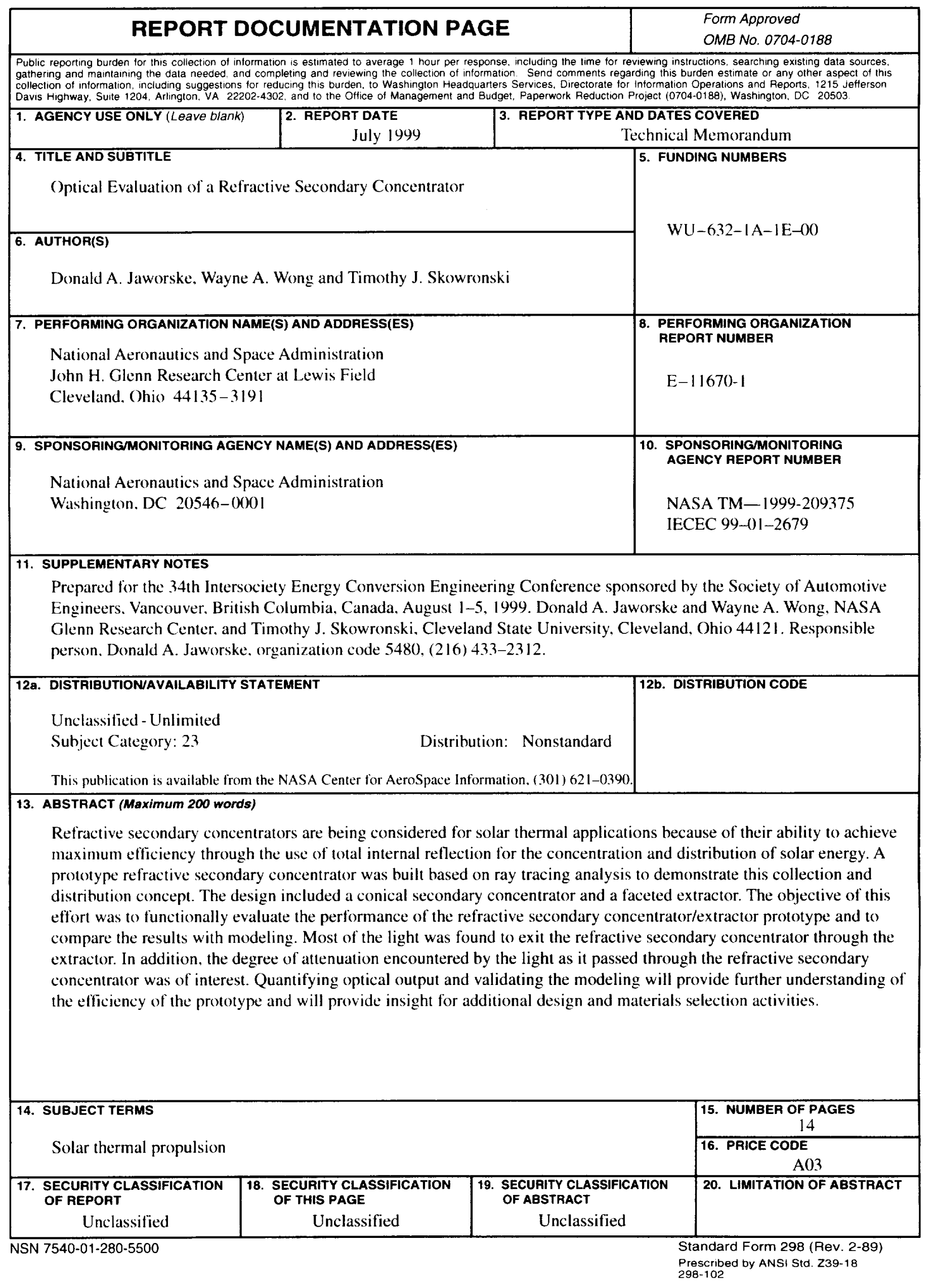

